\title{
Polyamines: An Essentially Regulatory Modulator of Plants to Abiotic Stress Tolerance: A Review
}

\author{
Fahmida Aktar ${ }^{1}$, Md. Shahidul Islam ${ }^{1}$, Md. Al-Amin Milon ${ }^{1}$, Nahidul Islam $^{1}$ and Md. Azizul Islam ${ }^{1, *}$ \\ ${ }^{1}$ Department of Biotechnology and Genetic Engineering, Faculty of Biological Sciences, Islamic University, Kushtia-7003, \\ Bangladesh \\ ${ }^{*}$ Corresponding author's email: azizbge.iu [AT] gmail.com
}

\begin{abstract}
Environmental stimuli including abiotic stresses, most notably salinity, drought, and cold stress greatly impact the growth, development, productivity, and disposal of plants worldwide. It has been calculated that two-thirds of the sustainable crop productivity are routinely facing a great challenge due to the unfavorable environmental factors by reducing the potential yield. There is a substantial amount of evidence to support that polyamines (PAs) are highly accumulated under abiotic stresses. PAs have a low molecular weight, are positively charged at physiological $\mathrm{pH}$, and have a high affinity for nucleic acids and membrane phospholipids, which are found in all living cells. The most frequently used putrescine (Put), spermidine (Spm), and spermine (Spm) are wellknown PAs that play a significant role in cell growth and development, leaf senescence, embryogenesis, and modulating plant tolerance to a wide range of abiotic stresses. These studies are highlights to dissever the action of polyamines mechanism in order to develop new strategies to enhance plant continuity in unfavorable environmental conditions.
\end{abstract}

Keywords- Abiotic stress, polyamines, stress tolerance, ROS, Transgenic plants

\section{INTRODUCTION}

Plants are sessile, sensitive species that are subjected to a wide range of environmental stresses over the course of their lives. Environmental stresses have a negative impact on plant growth and productivity. Soil salinity, exposure to extremes of temperature, and drought are the major abiotic stress factors that restrict crop productivity and plant development [1]. Environmental stresses have hampered crop cultivation and reduced average yields for the major crop plants by more than half in many regions of the world throughout the last decade [2]. It is projected that the world's population will reach around 10 billion by 2050 and this will experience serious food scarcity. The main challenge of modern agriculture is probably the maintenance of crops yielding under adverse environmental stresses. Plants can detect changes in their environment, react to them, adapt, and survive. Cellular, hormonal, biochemical, and molecular changes arise in response to stress. Different stresses trigger the expression of a wide range of genes in a large number of plants [3]. Biological evidence shows that plants use an overall and preserved mechanism for responding to abiotic stress. Natural signal transduction pathways in Arabidopsis thaliana can work together to resolve stress conditions [4]. The main PAs are putrescine, spermidine and spermine are small aliphatic nitrogenous compounds with positively charged molecules that are found in all over the living cell. This property allows PAs to interact with negatively charged macromolecules as DNA and RNA, proteins and phospholipids and involved in many processes of plant growth and development and the regulation of physical and chemical properties of membranes, cell wall stabilizing, as well as functions and modulation of enzyme activities [5]. The amount of free PAs in plant cells is determined by their transport, degradation, and conjugation, as well as their synthesis. A copper-containing enzyme Diamine oxidase (DAO) catalyzes at the elementary amino group of putrescine degradation, while a flavin-containing enzyme polyamine oxidase (PAO) catalyzes Spd and Spm oxidation as a secondary amino groups. Therefore, PAs can also be conjugated with the most common of small hydroxycinnamic acid molecules, such as proteins, antibiotics and phenol acids [6]. It has been noted that environmental stress resistance has increased in several plants after genetic transformation, with polyamine biosynthetic genes encoding agmatine deiminase (ADI), ADC, DAO, and ODC, 1-aminocyclopropane-1-carboxylic acid (ACC), SAMDC, SPDS, or SPMD [7]. It is noteworthy that SPDS [8], ADC [9], or SAMDC [10] overexpressing transgenic plants can tolerate many stresses, including salinity and drought, temperature and toxic to parquet. In this review, we will describe the biosynthesis and metabolism of PAs and identify current advances in polyamine mechanisms in plants under abiotic stress. 


\section{BIOSYNTHESIS OF PAs}

PAs are a class of phytohormones-including aliphatic amine compounds, mostly triamine-Spd, tetramine-Spm and its diamine compulsory precursors Put, which are omnipresent in every plant cell [11]. Put, Spd \& Spm are the major pathways for polyamines biosynthetic \& degradation (Figure 1).

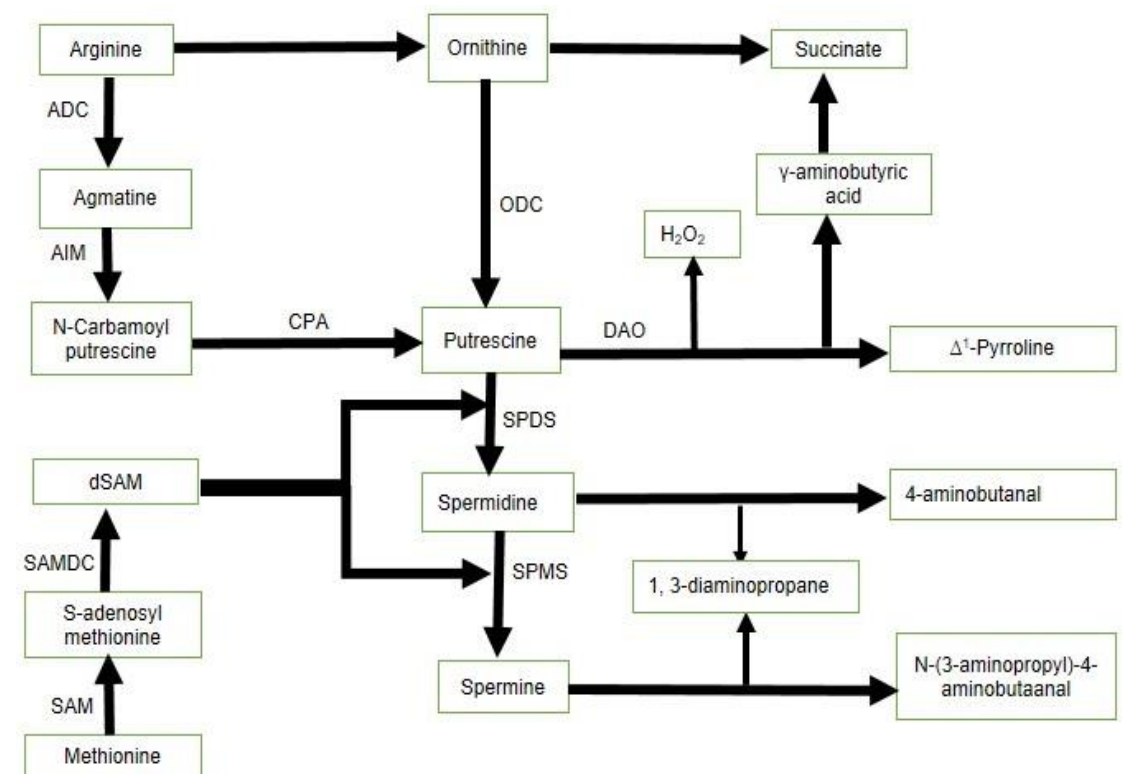

Figure 1 Polyamines biosynthesis pathway in plants abiotic stress. AIM: Agmatine iminohydrolase; ADC: Arginine decarboxylase; DAO: Diamine oxidase; ODC: Ornithine decarboxylase; SAMDC: S-adenosylmethionine decarboxylase;

SAM: S-adenosylmethionine; dSAM: Decarboxylated S-adenosylmethionine, CPA: $N$-Carbamoylputrescine amidohydrolase; $\mathrm{H}_{2} \mathrm{O}_{2}$ : Hydrogen peroxide; SPDS: Spd synthase; SPMD: Spermine synthase

The number of positive charges in the physiological pH of the cell is the key difference between Put, Spd, and Spm. Decarboxylated S- adenosylmethionine (dcSAM) which provides an amino propyl mobility for Put has been formed by S-adenosylmethionine (SAM) and is synthesized by SPDS via its addition to this put. While, the ADC generates the diamine Put via the agmatine (Agm) pathway or ODC pathway [11]. CPA and ADI are two enzymes necessary for the conversion of Agm to Put. Spd acts for the manufacture of the higher polyamine Spm as a substrate. Put catabolizes DAOs in a reaction that transforms Put to 1-pyrroline and produces ammonia and $\mathrm{H}_{2} \mathrm{O}_{2}$ (Hydrogen peroxide) as byproducts. Under normal and stress conditions, DAOs are favorably localized in plant cell walls, and hydrogen peroxide produced by Put catabolism can play a role in lignification and cross-linking reactions. In comparison to ADC1, which is expressed constitutively in all plant tissues, ADC2 is primarily responsible for abiotic stresses such as drought and wounding [12]. After all, plants with genes for PA biosynthesis can only be found in Arabidopsis, besides ODC [13]. The Arabidopsis genome is devoid of ODC, which has been discovered in many experiments.

\section{METABOLISM OF PAs}

The main enzymes of the polyamines biosynthesis pathway are regulated by a developmental control and complex metabolic process, all of which are needed for efficient cell metabolism regulation (Figure 2). Since then, scientists have been examining differences in PA and in plants subjected to single or multiple stresses [14]. The Expression levels of ADC (ADC1 \& ADC2) has been identified in Arabidopsis during environmental stresses. ADC2 exposure actively induces some abiotic pressures, while ADC1 exposure is primarily influenced by cold [15]. SPMS has a similar expression pattern to ADC2, resulting in polyamine aggregation in the presence of dehydration and elevated salinity [16]. Cold activates both SAMDC1 and SAMDC2 genes, while SAMDC2 is activated only by salt stress. The promoters of these genes contain ABA responsive elements [17]. The PA biosynthesis enzymes are regulated by transcription, translation, and post-translational processing. It is noteworthy that these enzymes first are synthesized as an inactive precursor (pre-enzyme), which is subsequently translated into mature enzymes. The level of PA in plant organs could be influenced by its degradation under stress conditions, and cellular PA is managed by the catabolic pathway [18]. DAO's function is to transform Put into pyrroline, ammonia, and $\mathrm{H}_{2} \mathrm{O}_{2}$. PA transport in the apoplastic was shown to be induced by salt stress \& stress hormone ABA therapy [19]. If PA catabolizes $\mathrm{H}_{2} \mathrm{O}_{2}$ from apoplastic stress can also lead to programmed cell death (PCD). Recent studies say the increased oxidation of PA in the apoplast either altered the balance 
between different reactive oxygen species (ROS) [20] or led to GABA ( $\gamma$-aminobutyric acid) accumulation by stimulating $\mathrm{CuAO}$ activities to support plant development in conditions of salt tension.

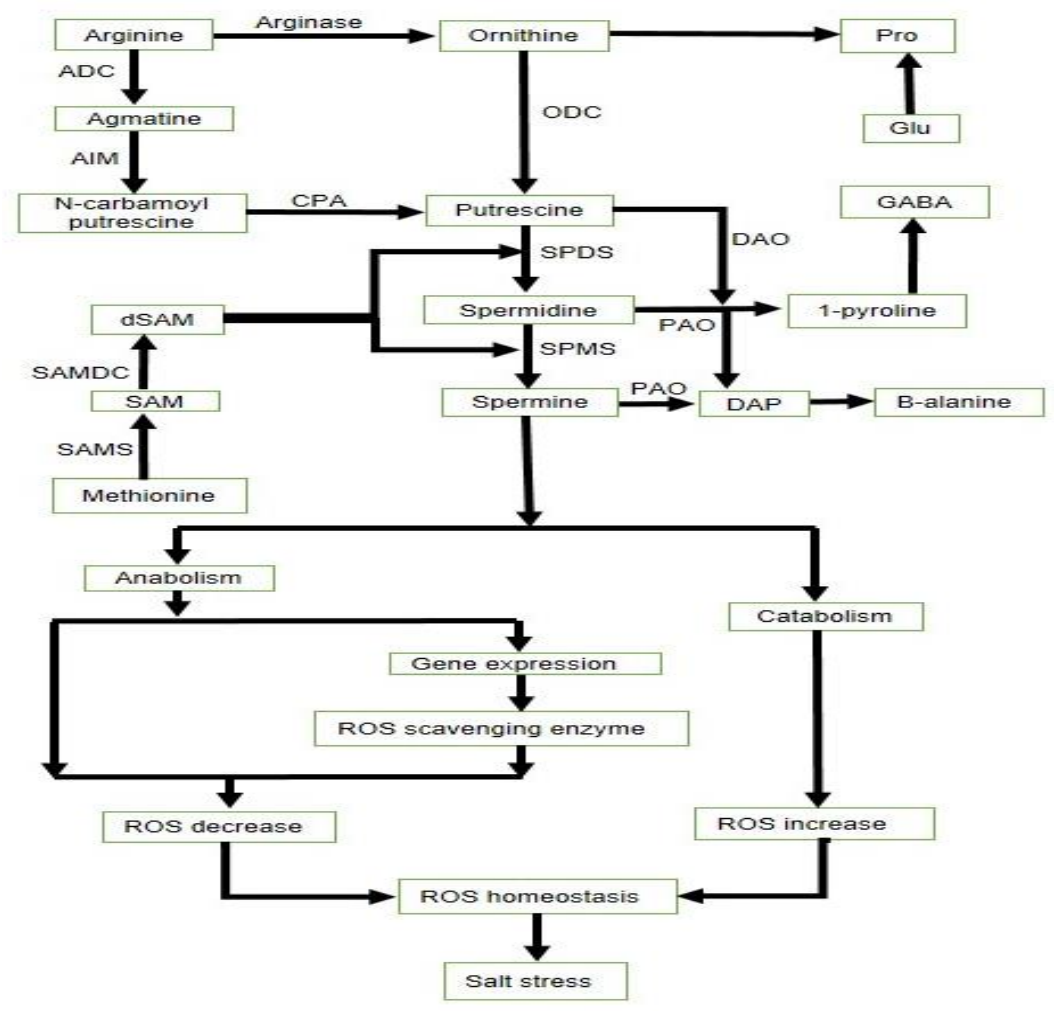

Figure 2 PAs metabolism in abiotic stress plant. PAO: Polyamine oxidase; CPA: $N$-carbamoylputrescine amidohydrolase (CPA), GABA: $\gamma$-aminobutyric acid; DAP: Diaminopropane; Pro: Proline; Glu: Glutamate; ROS: Reactive oxygen species

\section{SALINITY STRESS}

The salinization of soils is one of the most important types of land erosion. Nearly every continent has salt soils. But in the arid and semi-arid regions of the world, the main problem with salinity is that evapotranspiration is not sufficient to release soluble salts from the root area [21]. A high concentration of salt disrupts cell membrane, enzyme activity and photosynthetic appliance operation [22]. Plants respond to shift in the unfavorable atmosphere of low molecular osmolytes like proline. PAs concentrations on various rice varieties have shown that salt-resistant rice varieties sustain a high PAs level, e.g. Spd \& Spm, whereas salt-sensitive rice cultivars maintain only a high Put level. Salt resistant cultivars AU1, Co43, \& CSCl maintained high levels of Spd \& Spm, but in the growth stages studied where plants were subjected to salinity, the contents of Put were not altered significantly. In the salt system of the sensitive rice cultivars Co36, CSC2, GR3, IR20, TKM4 and TKM 9, excessively accumulated Spd \& Spm was related to the salt susceptibility in saline conditions. In almost all the plant species analyzed, however, salinity caused a major rise in Spd \& Spm. This meant that the Put pool was aimed at synthesizing Spd \& Spm. The Put ratio $(\mathrm{Spd}+\mathrm{Spm})$, which corresponds to the notion of a defensive effect of higher PAs (Spd \& Spm) against salt tension, has increased with the salinity ratio. In addition, Mansour and Al-Mutawa reported low Spd or Spm concentrations, whereas Put was successful, cellular alterations induced by $\mathrm{NaCl}$ in wheat roots [23]. El-Shintinawy et al. showed that the salinity of the Spm \& Spd has significantly increased the aggregation of Put in wheat cultivars [24]. But the OsSPDS2 (SPDS gene) mRNA in ricin plants was not greatly altered by $200 \mathrm{mM} \mathrm{NaCl}$ [25].

\section{DROUGHT STRESS}

During drought stress, the polyamine's degradation agent GABA levels were significantly higher. Put \& Spd produce GABA as a byproduct of D1-pyrroline degradation [18]. Those certain metabolic pathways can also affect GABA concentrations [26]. Under drought conditions, the pool size of arginine, the key substrate for polyamine biosynthesis, showing that this pathway was not substrate constrained. Ornithine, another potential substrate, was raised in half of the 
cultivars and reduced in the other half. Arginine is also a substrate in the biosynthesis of proline [27], where it is transformed to ornithine by arginase. Catabolism of polyamines not only decreases cellular polyamines, but also contributes to essential physiological processes through its enzymes and items. Do et al. found that under drought stress, Put and Spd levels were lower, while Spm levels go up in all cultivars studied, rendering it the most abundant polyamine [28]. The amount of Spm accumulated in rice matches previous findings [29]. Moreover, owing to the conversion of Put to Spd \& Spm, transgenic rice overexpressing the Datura stramonium ADC gene showed improved drought tolerance [30]. Rice plants that express SAMDC from D. Stramonium have shown unchanged amounts of Spm at Spd expense, which have caused drought-like symptoms as wild, but no bust-related recovery again watered conditions [31]. Boucherau et al. suggested that Put should normally accumulate in susceptible organisms under stress conditions and Spd \& Spm should not be accumulated [32]. The ADC mediated Put aggregation is believed to be the main enzyme responsible for abiotic stress [33].

\section{TEMPERATURE / HEAT STRESS}

Temperature stress creates problems, so it's crucial to find out how plants deal with it. The suboptimal temperature stress is any growth or induced metabolic, cellular or tissue injury which reduces the genetically defined yield potential, directly due to thermal temperature exposure up or down for suitable biochemical and physiologic action, or morphological development. Significant increases of free \& conjugated PAs \& long-chained PA and a high accumulation of PAOs \& PA biosynthesizing ADC during heat stress were reported in heat-tolerant cotton and rice [34]. A rise in ADC \& PAO activities was identified in the heat tolerant rice cultivars. The adaptation mechanism is now well accepted as also important to the photosynthesis apparatus. During plant exposure to various environmental stress like salt tolerance, UV-B, ozone, heavy metal, water stress or chilling, difference in thylakoid-associated PAs has actually been observed. It has been found that high $\mathrm{CO}_{2}$ concentrations increased the thylakoid bond Put [35]. However, the thylakoid-associated Spm was increased by UV rays [36]. Exogenous addition of Put during salt stress has been shown to improve the photochemical performance of PS-ll in several studies [37]. Furthermore, using PAs during stress-induced senescence avoided chlorophyll degradation and maintained the structure of the thylakoid membranes.

\section{COLD STRESS}

As heat stress, cold stress can be fatal for plants. The water inside a cell expands when it freezes just like water transforms to ice. This can rupture the cell membrane, resulting in cell death. Plants react to cold temperatures by triggering metabolic pathways that protect their cells from the cold and freezing. In reaction to cold stress, rice plants have been shown to serve a key role in the PA biosynthetic pathway [38]. Cold-tolerant varieties have been shown to have greater intrinsic levels of polyamines in responding to low temperatures than non-tolerant varieties. Therefore, free Put accumulation was observed in Arabidopsis within 24 hours of cold exposure but there were no variations in Spd levels [39]. Quantitative expression analysis of the ADC1 and ADC2 genes reveals that transcription is triggered as quickly as 30 minutes after exposure to cold, while ADC1 having a greater amplitude. The presence of CRT/DEE (Crepeat / Dehydration - Sensitive Element) in the promoter of ADC1, which confers responsiveness to low temperature, drought and salinity can mediate the early and temporary upregulation of ADC1 towards cold stress [15]. It was discovered that increased ADC1 \& ADC2 activists resulted in the accumulation of Put during cold stress. Vogel et al. (2005) showed that Zat12, a cold-inducible $\mathrm{C}_{2} \mathrm{H}_{2}$ zing finger transcription factor, has been upregulated in cold stress [40]. As a result, zeaxanthin is activated by Put, resulting in the accumulation of ABA. Most stress sensitive elements include ABA responsive elements (ABRE), which are further triggered, resulting in the development of defensive metabolites and cold acclimation [40].

\section{METAL STRESS}

Heavy metal pollution and persistence in water, soil, and air as a result of various factors is now a global environmental concern that has a significant impact on rice growth and quality. Plant growth, metabolism, and development are all harmed by heavy metals deposited in land [41]. As a result, the transport of heavy metals from soil to plants with commercial agricultural value, such as rice, is of great concern because it could contribute to biomagnification via the ecosystem that could have a number of negative consequences for consumers [41]. Metal toxicity in plants can be caused by metals binding to sulfhydryl groups of proteins, inhibiting enzyme activity or altering protein structure, as well as stimulating the formation of ROS that cause macromolecule oxidation and oxidative stress [42]. Metal uptake is sometimes limited by its bioavailability in the soil, in addition to genetic variation. The availability of metal ions such as $\mathrm{Fe}, \mathrm{Zn}$, and $\mathrm{Cd}$ for plant uptake is largely determined by the redox potential of the soil. When sunflower or wheat leaf discs treated with $\mathrm{Cd}^{2+}$ or $\mathrm{Cu}^{2+}$, PA metabolism was affected differently, and increased ADC and ODC were both attributed to Put levels in $0.5 \mathrm{mM}$ cadmium treated wheat leaf discs [43]. In comparison to Lin \& Kao, who found that Put accumulation was related to ADC activity in copper-treated rice leaves, ODC appeared to be the key enzyme responsible for Put biosynthesis under copper treatment [44]. Copper reduced ADC function, yet had no effect on ODC or DAO activity, suggesting that a decline in ADC interaction is to blame for the lower Put levels observed [43]. Extracellular inclusion of Spd or Spm totally altered the metals-induced TBARS increase in sunflower leaf discs treated with cadmium and copper in terms of antioxidative actions of PAs. After $\mathrm{Cd}^{2+}$ or $\mathrm{Cu}^{2+}$ treatments, $1 \mathrm{mM} \mathrm{Spd} \mathrm{or} \mathrm{Spm}$ 
applied to the metals restored the reduced glutathione reductase (GR) and superoxide dismutase (SOD) activities [45].

\section{OZONE STRESS}

Plants across the globe, including food crops and plants in natural habitats, are seriously harmed by ozone. Plants are harmed by ozone because it enters leaf openings called stomata and oxidizes (burns) plant tissue during respiration. This harms the plant's leaves and reduces its chances of survival. The most phytotoxic gas pollutant in the atmosphere is ozone. It reaches the plant via the stomata, causing a number of toxic effects such as membrane defective, toxic compound production, $\mathrm{CO}_{2}$ assimilation inhibition, growth inhibition, early senescence, and cell death [46]. Severe presence of high ozone may cause chlorosis and necrotic lesions in the foliage of ozone-sensitive crops at the molecular level. The emergence of ROS such as $\mathrm{O}_{2}$ and $\mathrm{H}_{2} \mathrm{O}_{2}$ by ozone degradation in the apoplast has been proposed as a mechanism for ozone-induced damage [47]. Plants can also increase PAs concentrations in addition to ozone toxicity, and the function of plant PAs in protecting towards ozone impairment and ozone imitative oxidative stress has been well established, though the exact mode of action remains unclear [48]. When the hypersensitive cultivars $B e \mathrm{~W}_{3}$ were present, only a slight increase or decrease in Put was observed at a later stage. In ozone-treated tobacco cultivars Bel B, the aggregation of unrestricted and covalently linked Put was followed by an increase in ADC activity. Free Put built up in the unharmed tissue of Bel $\mathrm{W}_{3} \&$ Bel $\mathrm{B}$ cultivars in tobacco plants treated with ozone. This aggregation triggered a temporary rise in ADC and ODC related activities. The ozone-induced programmed cell death reaction in Bel $\mathrm{W}_{3}$ plants includes the buildup of associated Put. Ozone tolerance was not connected to ozone-induced free Put synthesis in Bel B plants, which tend to have problems with signal transduction pathways that regulate cellular redox state [48].

\section{UV TREATMENT}

Increased UV-B radiation (280-315 nm) in the biosphere can cause an increase in ROS formation in plant tissues, as well as changes in antioxidant enzyme activities and PAs content causes oxidative stress. Complete free PAs decreased rapidly with respect to UV-B radiation in Phaseolus vulgaris plants (primarily because of a decrease in Put content), which was linked to UV-B mediated chlorophyll depletion [49]. An increase in PAs bound, where cell wall attached to phenolics, may explain a decrease in PAs free levels. Zacchini \& de Agazio discovered that Put content increased in upper layers of tobacco callus after 6 hours of UV-C irradiation, but decreased after 24 and 48 hours, though it remained statistically higher than controls [50]. There were no variations between UV-irradiated calli and controls in Spd and Spm, and UV-treatment had no effect on lower layers. UV-B rays were responsible for a dose-dependent decrease in leaves field, leaf dry matter, and plant length in cucumber plants, as well as a substantial increase in Put, Spd, \& Spm concentrations [51]. According to the scientists, PAs aggregation in cucumber leafage is a stress-response process to UVB radiation. Lutz et al. stated that an increase in PAs, particularly Put in thylakoid membranes of the Bel B variety, is one of the primary protective mechanisms to the photosynthetic apparatus against UV-B rays in daylight inducers with natural wavering of simulated UV-B [52]. Furthermore, the inability to increase Put levels in thylakoid membranes was due to Bel $\mathrm{W}_{3}$ cultivars' UV-B sensitivity though it is sensitive to ozone. The PAs level decreased after prolonged UV-B exposure and the development of other secondary responses.

\section{CHILLING STRESS}

Chilling, a natural environmental stress in nature, may have a direct impact on biological mechanisms of chloroplasts. It for starters, will modify enzyme activity and lipid membrane condition in chloroplasts. The energy of photosynthesis then reduces, resulting in a high level of ROS production. In the one side, residual ROS will destroy the lipid membrane of the chloroplast; in the contrary, ROS may act as a stress signal, altering gene expression in both the nucleus and the chloroplast to help regenerate weakened proteins, control lipid homeostrains, and encourage plant tolerance to low temperatures. There are just a few accounts of PAs being involved in chilling stress [53]. It procedure has a different effect on PAs quality in leaves in cold-tolerant and cold-sensitive cucumber cultivars. Furthermore, pre-treating the cold sensitive cultivar with PAs reduced chilling damage. Increased ODC activity may explain the rise in Put, Spd, and Spm in the freezing tolerant varieties throughout chilling. Put elevation was the highest, but it was only transient, and its reduction seemed to coincide with the advent of flower and pod abscission in stressed plants. In stressed plants, electrolyte leakage increased when cellular respiration decreased, and Put reversed these patterns when it was applied exogenously to stress plants [54]. Even though their PAs pools dramatically decreased during chilling, adding Spd to the growth medium prior to the cold treatment increased Spd content in all organs and resulted in higher cold tolerance in cucumbers [55]. As a result, PAs deposition in reaction to cold could be avoided in chilling injury if PAs levels were elevated prior to chilling exposure and remained high during the stress and stress procedure. Imai et al. discovered that a new SPDS gene, OsSPDS2 of rice, is implicated in the chilling reaction in roots [25].

\section{WOUNDING STRESS}

Plants are continuously exposed to elements such as mechanical pressures from the atmosphere and herbivores, which cause injuring and enable pathogenic microbes to invade. Wounding provides bacteria with nutrition and facilitates their entrance into tissue and inflammation. MAMPs (microbe-associated molecular patterns) and DAMPs (Damage- 
associated molecular patterns) cause defense mechanisms, implying that plants restrict both injury and pathogens in a way analogous [56]. Plants respond to wounds with both constitutive structures that prevent opportunistic microorganisms from entering the tissue, such as epicuticular films and wax crystals, along with wound-induced responses that, with the exception of the constitutive defense, are intensely expensive and thus controlled and triggered [57].

\section{OXIDATIVE STRESS}

Oxidative stress is due to a disparity between the ROS synthesis \& the amelioration of free radicals by antioxidants, leading to disruption of cellular components such as lipids, nucleic acids, metabolites, and proteins, eventually causing plant cell death. PAs were being studied as antioxidants over the last few years under a number of unfavorable environmental circumstances, yet their specific function as antioxidants is still being debated [58]. PAs have an antioxidative effect caused by their anion and cation binding properties, which include a radical scavenging mechanism and the capacity to suppress both lipid peroxidation and metal -catalyzed oxidative reactions. Spermine, Spermidine, and Putrescine all aid in lowering the amount of superoxide radicals formed by senescent plant cells. Conversely, PA catabolism releases $\mathrm{H}_{2} \mathrm{O}_{2}$, a triggering molecule which can activate an antioxidative defense mechanism by entering the stress signal transduction chain, and it can also serve as a prooxidant agent. In reaction to stress, phenylpropanoid-PA complexes may serve as antioxidants toward reactive nitrogen species (RNS) and ROS [59]. Under abiotic stress, ROS, distinguished by the aggregation of toxic molecules $\mathrm{O}^{2-}, \mathrm{H}_{2} \mathrm{O}_{2}$, and $\mathrm{OH}^{-}$in tissues, is a typical plant reaction worthy of destroying plant cell membranes and macromolecules [60]. PAO's development of ROS is intimately associated with PA catabolic reactions that are linked to plant protection and abiotic stress defenses [61].

\section{PA BIOSYNTHETIC GENES IN TRANSGENIC PLANTS}

Transgenic plants were generated (Table 1) by the ADC gene of oats and datura plants, with increased Put, Spd, and Spm accumulation in the plants are more resistant to salt and drought [62]. Transgenic A. thaliana noted both the deposition of Put and an increased resistance to dehydration, freezing and salt stress [9]. The overexpressed ADC of $A$. thaliana of exhibited higher Put synthesis and enhanced osmotic, drought, and cold stress tolerance [63]. SAMDC included transgenic rice plants increased salt stress [10] The component of SAMDC yeast gene, which was transgenic tomato plants, accumulated 1.7 to 2.4 times the levels of Spd and Spm, and showed resistance to this stress compared with the wild-type plants under temperature stress $\left(38^{\circ} \mathrm{C}\right.$ for $\left.1 \mathrm{~h}\right)$. SAMDC overexpression in tobacco resulted in higher Put and Spd levels, resulting in salt and osmotic stress tolerance [64]. Increased titer of the Spd gene and increased tolerances to various abiotic stresses relative to non-transgenic plants demonstrated over-expression of SPDS gene on the transgenic lines A. thaliana and sweet potato (Ipoeean batatatus) and pear (Pyrus communis). The excessive expression of the SPDS gene in pear has shown altered PA levels and enhanced abiotic stress tolerance. PAs were shown to be molecular chaperones and essential protecting elements in dryness, salinity and temperature stress resistance through functional studies of stress tolerance genes [65]. Genetic engineering of multiple metabolic pads of many solutes consistent with abiotic stress tolerance such as proline or glyceinbetain is also essential [66]. Abiotic stress tolerance is a dynamic process that involves the adaption, perpetuation, and receptive attitude of stress tolerance by the regulation of multiple metabolic pathways through cyclical crosstalk [67]. Both of these studies supports the opinion that extending PA biosynthesis may be an appropriate system for transgenic science, taking into account plant stress and improving the resistance of stress-sensitive plants toward antagonistic environmental conditions. The overexpression of heterologous characteristics in any event provides little evidence on the importance, in the case of salt and dry tension, of endogenous PA amalgamation and endogenous chemicals. The endogenous properties of the PA digestive activity can be understood by studies of freaks with reduced chemical movement in different pressure reactions.

Table 1 Polyamines accumulated by transgenic plants under abiotic stress tolerance.

\begin{tabular}{c|l|l|l|l|l}
\hline $\begin{array}{c}\text { PA } \\
\text { genes }\end{array}$ & Transgenic plants & \multicolumn{1}{c}{ Source } & $\begin{array}{c}\text { Accumulated } \\
\text { PA }\end{array}$ & Tolerance & References \\
\hline \multirow{4}{*}{ ADC } & Oryza sativa & Avena sativa & Put & Salinity & {$[68]$} \\
& Oryza sativa & Datura Stramonium & Put, Spd, Spm & drought & {$[30]$} \\
& Arabidopsis thaliana & Poncirus trifoliata & Put & Osmotic, drought, cold & {$[63]$} \\
& Arabidopsis thaliana & Avena sativa & Put & Drought, freezing & {$[9]$} \\
\hline \multirow{4}{*}{ SAMDC } & Oryza sativa & Triticum Turgidum & Spd, Spm & Salt & {$[10]$} \\
& Oryza sativa & Dianthus stramonium & Spd, Spm & Drought & {$[31]$} \\
& Nicotiana tabacum & Homo sapiens & Put, Spd & Salt, drought & {$[64]$} \\
& Solanum lycopersixcon & Saccharomyces & Spd, Spm & Heat, oxidative & {$[69]$} \\
& & cerevisiae & & & \\
\hline \multirow{2}{*}{ SPDS-1 } & Arabidopsis thaliana & Cucurbita ficifolia & Spd & Chilling, salt, drought & {$[8]$} \\
& Puyrus communis & Malus domestica & Spd & Heavy metal & {$[70]$} \\
\hline
\end{tabular}




\section{CONCLUSIONS AND FUTURE PROSPECTS}

Along with this audit, it stays clear that PAs are regular intensifies worried about plant reactions to a few sorts of abiotic pushes however numerous openings stay behind the striking advances in the field announced in the last ten years. Ecological difficulties from different sources "turn on" a limitless number of reactions in regards to PAs digestion. Nowadays, the availability of key genes involved in the biosynthetic pathways and catabolic processes have made possible to shed light on PA metabolism using diverse molecular strategies, especially through transgenic approaches and the use of mutants of PAs metabolism. Nonetheless, PAs limitation inside the cells, transport instruments, proteins engaged with PAs conveyance and the guideline of catabolic cycles at the transcriptional, translational and posttranscriptional level are key focuses that should be explained. It ought to be remember the need to relate PA digestion and physiology with plant development controllers from a practical perspective. A substantial amount of data analysis show that PA biosynthesis genes plays a vital role in environmental stress tolerance, and could be exploited in genetic engineering programs to produce stress-tolerant plants. Future strategies should be developed on the basis of a comprehensive knowledge-based PA metabolism, which includes PA biosynthetic genes and their control by stressinducible promoters and transcription factors of various types. A Comprehensive View of genetic engineering of PA biosynthetic genes, a biotechnology approach, may be critical in solving future problems involving environmental stressresistant plants using transgenic strategies [3].

\section{ACKNOWLEDGEMENT}

The authors are grateful to the Department of Biotechnology and Genetic Engineering, Islamic University Kushtia-7003, Bangladesh for research facilities and other logistic supports.

\section{REFERENCES}

[1] Tuteja N. and Sopory S.K., "Chemical signaling under abiotic stress environment in plants", Plant Signal Beh, vol. 3, pp.525-36, 2008.

[2] Mahajan S. and Tuteja N., "Cold, salinity and drought stresses: An overview", Arch Biochem Biophy, vol. 444, pp.139-58, 2005.

[3] Sanghera G.S., Wani S.H., Hussain W., and Singh N.B., "Engineering cold stress tolerance in crop plants", Curr Genomics, vol. 12, pp.30-43, 2011.

[4] Wang W., Vinocur B., and Altman A., "Plant responses to drought, salinity and extreme temperature: towards genetic engineering for stress tolerance", Planta, vol. 218, pp.1-14, 2003.

[5] Zhao H. and Yang H., "Exogenous polyamines alleviate the lipid peroxidation induced by cadmium chloride stress in Malus hupehensis Rehd", Sci Hortic, vol. 116, pp.442-7, 2008.

[6] Martin-Tanguy J., "Metabolism and function of polyamines in plants: recent development (new approaches)", Plant Growth Regul, vol. 34, pp.135-148, 2001.

[7] Liu J-H., Kitashiba H., Wang J., Ban Y., and Moriguchi T., "Polyamines and their ability to provide environmental stress tolerance to plants", Plant Biotechnol, vol. 24, pp.117-26, 2007.

[8] Kasukabe Y., He L., Nada K., Misawa S., Ihara I., and Tachibana S., "Overexpression of spermidine synthase enhances tolerance to multiple environmental stresses \& up-regulates the expression of various stress regulated gene in transgenic Arabidopsis thaliana”, Plant Cell Physiol, vol. 45, pp.712-722, 2004.

[9] Alet A.I., Sanchez D.H., Cuevas J.C., Valle S.D., Altabella T., Tiburcio A.F., Marco F., Ferrando A., Espasandin F.D., González M.E., Ruiz O.A., and Carrasco P., " Putrescine accumulation in Arabidopsis thaliana transgenic lines enhances tolerance to dehydration \& freezing stress", Plant Signal Behav, vol. 6, pp.278-286, 2011.

[10] Roy M. and Wu R., "Overexpression of S-adenosylmethionine decarboxylase gene in rice increases polyamine level \& enhances sodium chloride stress tolerance”, Plant Sci, vol. 163, pp.987-92, 2002.

[11] Sarvajeet S.G. and Narendra T., "Polyamines \& abiotic stress tolerance in plants", Plant Signaling \& Behavior, vol. 5, pp.126-33, 2010.

[12] Perez-Amador M.A., Leon J., Green P.J., and Carbonell J., "Induction of the Arginine decarboxylase ADC2 gene provides evidence for the involvement of polyamines in the wound response in Arabidopsis", Plant Physiol, vol. 130, pp.1454-63, 2002.

[13] Hanfrey C., Sommer S., Mayer M.J., Burtin D., and Michael A.J., “Arabidopsis polyamine biosynthesis: absence of ornithine decarboxylase and the mechanism of arginine decarboxylase activity”, Plant Journal, vol. 27, pp.551-560, 2001.

[14] Liu Ji-H., Nada K., Honda C., Kitashiba H., Wen X.P., Pang X.M., and Moriguchi T., "Polyamine biosynthesis of apple callus under salt stress: importance of Arginine decarboxylase pathway in stress response", J Exp Bot, vol. 57, pp.2589-99, 2006.

[15] Hummel I., Gouesbet G., El Amrani A., Ainouche A., and Couee I., "Characterization of the two arginine decarboxylase (polyamine biosynthesis) paralogues of the endemic subantarctic cruciferous species Pringlea 
antiscorbutica \& analysis of their differential expression during development and response to environment at stress", Gene, vol. 342, pp.199-209, 2004.

[16] Alcázar R., Marco F., Cuevas J.C., Patron M., Ferrando A., Carrasco P., Tiburcio A.F., and Altabella T., "Involvement of polyamines in plant response to abiotic stress", Biotechnol Lett, 2006; vol. 28, pp.1867-1876, 2006.

[17] Mukherjee K., Choudhury A.R., Gupta B., Gupta S., and Sengupta D.N., "An ABRE-binding factor, OSBZ8, is highly expressed in salt tolerant cultivars than in salt sensitive cultivars of indica rice", BMC Plant Biol, vol. 6, pp.18, 2006.

[18] Bagni N. and Tassoni A., "Biosynthesis, oxidation \& conjugation of aliphatic polyamines in higher plants", Amino Acids, vol. 20, pp.301-317, 2001.

[19] Yoda H., Hamaguchi R., and Sano H., "Induction of hypersensitive cell death by hydrogen peroxide produced through polyamine degradation in tobacco plants", Plant Physiol, vol. 132, pp.1973-1981, 2003.

[20] Rodriguez A.A., Maiale S.J., Menendez A.B., and Ruiz O.A., "Polyamine oxidase activity contributes to sustain maize leaf elongation under saline stress", J Exp Bot, vol. 60, pp.4249-4262, 2009.

[21] Miller G., Shulavev V., and Miller R., " Reactive oxygen signaling \& abiotic stress”, Plant Physiol, vol. 133, pp.481-489, 2008.

[22] Islam M.A., Maitra P., Biswas S.K., and Faruquee H.M., "Actions of polyamine on abiotic stresses in rice", IJSER, vol. 9, pp.73-80, 2018.

[23] Mansour M.M.F., and Al-Mutawa M.M., "Stabilization of plasma membrane by polyamines against salt stress", Cytobios, vol. 100, pp.7-17, 1999.

[24]El-Shintinawy F., "Photosynthesis in two wheat cultivars differing in salt susceptibility", Photosynthetica, vol. 38, pp.615-620, 2000.

[25] Imai R., Ali A., Pramanik M.H.R., Nakaninami K., Sentoku N., and Kato H., "A distinctive class of spermidine synthase is involved in chilling response in rice", J Plant Physiol, vol. 161, pp.883-886, 2004.

[26] Fait A., Fromm H., Walter D., Galili G., and Fernie A.R., "Highway or byway; the metabolic role of the GABA shunt in plants", Trends Plant Sci, vol. 13, pp.14-19, 2008.

[27] Hsu S.Y., Hsu Y.T., and Kao C.H., "The effect of polyethylene glycol on proline accumulation in rice leaves", Biol Plant, vol. 46, pp.73-78, 2003.

[28] DO P.T., Degenkolbe T., Erban A., Heyer G.A., Kopka J., Kohl K.I., Hincha D.K., and Zuther E., "Dissecting rice polyamine metabolism under controlled long-term drought stress", PLOS ONE, vol. 8, pp.e60325, 2013.

[29] Yang J., Zhang J., Liu K., Wang Z., and Liu L., "Involvement of polyamines in the drought resistance of rice", $J$ Exp Botany, vol. 58, pp.1545-1555, 2007.

[30] Capell T., Bassie L., and Christou P., "Modulation of the polyamine biosynthetic pathway in transgenic rice confers tolerance to drought stress", Proc Natl Acad Sci USA, vol. 101, pp.9909-9914, 2004.

[31] Peremarti A., Bassie L., Christou P., and Capell T., "Spermine facilitates recovery from drought but does not confer drought tolerance in transgenic rice plants expressing Datura stramonium S-adenosylmethionine decarboxylase", Plant Mol Biol, vol. 70, pp.253-264, 2009.

[32] Boucherau A., Aziz A., Larher F., and Martin-TanguyJ., "Polyamines \& environmental challenges; recent development", Plant Sci, vol.140, pp.103-125, 1999.

[33] Galston A.W., Kaur-Sawhney R., Altabella T., and Tiburcio A.F., "Plant polyamines in reproductive activity and response to abiotic stress", Bot Acta, vol. 110, pp.197-207, 1997.

[34] Cona A., Rea G., Angelini F., Federcio R., and Tavladoraki P., "Functions of amine oxidase in plant development \& defence", Trends Plant Sci, vol. 11, pp.80-88, 2006.

[35] Alcázar R., Marco F., Cuevas J.C., Patron M., Ferrando A., Carrasco P., Tiburcio A.F., and Altabella T., "Involvement of polyamines in plant response to abiotic stress", Biotechnol Lett, vol. 28, pp.1867-1876, 2006.

[36] Sfichi L., Ioannidis N., and Kotzabasis K., "Thylakoid-associated polyamines adjust the UV-B sensitivity of the photosynthetic apparatus by means of light- harvesting complex II changes", Photochem Photobiol, vol. 80, pp.499$506,2004$.

[37]Zhang R.H., Li J., Guo S.R., and Tezuka T., "Effects of exogenous putrescine on gas exchange characteristics \& chlorophyll fluorescence of NaCl-stressed cucumber seedlings", Photosynth Res, vol. 100, pp.155-162, 2009.

[38] Cook D., Flower S., Fiehn O., and Thomashow M.F., "A prominent role for the CBF cold response pathway in configuring the low temperature metabolome of Arabidopsis", Proc Natl Acad Sci, vol. 101, pp.15243-15248, 2004.

[39] Cuevas J.C., Lopez-Cobollo R., Alcazar R., Zarza X., Koncz C., Altabella T., Salinas J., Tiburcio A.F., and Ferrando A., " Putrescine is involved in Arabidopsis freezing tolerance \& cold acclimation by regulating abscisic acid levels in response to low temperature", Plant Physiol, vol. 148, pp.1094-1105, 2008.

[40] Vogel J.T., Zarka D.G., Van Buskirk H.A., Fowler S.G., and Thomashow M.F., "Roles of the CBF2 \& ZAT12 transcription factors in configuring the low temperature transcritome of Arabidopsis", Plant J, vol. 41, pp.195-211, 2005.

[41] Anitha M. and Kavitha S., "Heavy metal \& mineral element-induced abiotic stress in rice plant", DOI:10.5772/intechopen.76080, 2018. 
[42] Sandalio L.M., Dalurzo H.C., Gomez M., Romero Puertas M.C., and del Rio L.A., "Cadmium-induced changes in the growth \& oxidative metabolism of pea plants", J Exp Bot, vol. 52, pp.2115-2126, 2001.

[43] Groppa M.D., Benavides M.P., and ML Tomaro M.L., "Polyamine metabolism in sunflower \& wheat leaf discs under cadmium or copper stress", Plant Sci, vol. 164, pp.293-299, 2003.

[44] Lin C.H. and Kao C.H., "Excess copper induces an accumulation of putrescine in rice leaves", Bot Bull Acad Sin, vol. 40, pp.213-218, 1999.

[45] Groppa M.D., Tomaro M.L., and Benavides M.P., "Polyamines as protectors against cadmium or copper-induced oxidative damage in sunflower leaf discs", Plant Sci, vol. 161, pp481-488, 2001.

[46] Rao M.V., Koch J.R., and Davis K.R., "Ozone: a tool for probing programmed cell death in plants", Plant Mol Biol, vol. 44, pp.345-358, 2000.

[47] Baier M., Kandlbinder A., Golldack D., and Dietz KJ., "Oxidative stress \& ozone: perception, signaling \& response", Plant cell Environ, vol. 28, pp.1012-1020, 2005.

[48] Van Buuren M.L., Guidi L., Fornale S., Ghetti F., Franceschetti M., Soldatini G.F., and Bagni N., “Ozone response mechanisms in tobacco: implications of polyamine metabolism”, New Phytol, 1vol. 56, pp.389-398, 2002.

[49] Smith J., Burrit D., and Bannister P., "Ultraviolet-B radiation leads to a reduction in free polyamines in Phaseolus vulgaris L", Plant Growth Regul, vol. 35, pp.289-294, 2001.

[50] Zacchini M. and de Agazio M., "Spread of oxidative damage \& antioxidative response through cell layers of tobacco callus after UV-C treatment", Plant Physiol Biochem, vol. 42, pp.445-450, 2004.

[51] An L.Z., Liu G.X., Zhang M.X., Chen T., Liu Y.H., Feng H.Y., Xu S.J., Qiang W.Y., and Wang X.L., “ Effect of enhanced UV-B radiation on polyamine content \& membrane permeability in cucumber leaves", Russ J Plant Physiol, vol. 51, pp.658-662, 2004.

[52] Lutz C., Navakoudis E., Seidlitz H.K., and Kotzabasis K., "Simulated solar irradiation with enhanced UV-B adjust plastid \& thylakoid associated polyamine changes for UV-B protection", Biochim Biophys Acta, vol. 1710, pp.24-33, 2005.

[53] Kim T.E., Kim S.K., Han T.J., Lee J.S., and Chang S.C., “ABA \& polyamines act independently in primary leaves of cold-stressed tomato (Lycoperscion esculentum)”, Physiol Plant, vol.115, pp.370-376, 2002.

[54] Nayyar H., "Putrescine increases floral retention, pod set \& seed yield in cold stressed chickpea", J Agron Crop Sci, vol. 191, pp.340-345, 2005.

[55]He L., Nada K., and Tachibana S., "Effects of Spermidine pretreatment through the roots on growth and photosynthesis of chilled Cucumber plants (Cucumis sativus L)”, J Jpn Soc Hort Sci, vol. 71, pp.490-498, 2002.

[56] Savatin D.V., Gramegna G., Modesti V., and Cervone F., "Wounding in the plant tissue: the defense of a dangerous passage", Frontiers in Plant Science, vol. 5, pp.1-11, 2014.

[57] Bonaventure G. and Baldwin T., "Transduction of wound \& herbivory signals in plastids", Common Integer Biol, vol. 3, pp.313-317, 2010.

[58] Chattopadhyay M.K., Tiwari B.S., Chattopadhyay G., Bose A., Sengupta D.N., and Ghosh B., "Protective role of exogenous polyamines on salinity stressed rice (Oryza sativa) plant", Physiol Plant, vol. 116, pp.192-199, 2002.

[59] Yamasaki H. and Cohen M.F., "NO signal at the crossroads: Polyamine induced nitric oxide synthesis in plants", Trends Plant Sci, vol. 11, pp.522-524, 2006.

[60] Apel H. and Kirt H., "Reactive oxygen species: metabolism, oxidative stress \& signal transduction", Annu Rev Plant Biol, vol. 55, pp.373-379, 2004.

[61] Roy P., Niyogi K., Sen Gupta D.N., and Ghosh B., "Spermidine treatment to rice seedlings recover salinity stressinduced damage of plasma membrane \& PM-bound H+ ATPase in salt tolerant \& salt sensitive rice cultivars", Plant Sci, vol. 168, pp.583-91, 2005.

[62] Bassie L., Zhu C., Romagosa I., Christou P., and Capell T., "Transgenic wheat plants expressing an oat arginine decarboxylase cDNA exhibit increases in polyamine content in vegetative tissue \& seeds", Mol Breed, vol. 22, pp.39-50, 2008.

[63] Wang J., Sun P.P., Chin C.L., Wang Y., Fu X.Z., and Liu J.H., "An arginine decarboxylase gene AtADC from Poncirus trifoliata confers abiotic stress tolerance \& promotes primary root growth in Arabidopsis", J Exp Bot, vol. 62, pp.2899-2914, 2011 b.

[64] Waie B. and Rajma M.V., "Effect of increased polyamine biosynthesis on stress response in transgenic tobacco by introduction of human S-adenosylmethionine gene", Plant Sci, vol. 164, pp.727-734, 2003.

[65] Tiburcio A.F., Wollenweber B., Zilberstein A., and Koncz C., “Abiotic stress tolerance”, Plant Sci, vol. 182, pp.1$2,2012$.

[66] Zhao L-L., Song S-Q., You C-X., Moriguchi T., and Hao Y-J., "Functional characterization of the apple MdSAMDC2 gene by ectopic promoter analysis \& overexpression in tobacco”, Biol Plant, vol. 54, pp.631-638, 2010.

[67] Xu Y., Shi G.X., Ding C.X., and Xu X.Y., "Polyamine metabolism \& physiological responses of Potamogeton crispus leaves under lead stress", Russ J Plant Physiol, vol.58, pp.460-6, 2011.

[68] Roy M., and We R., "Arginine decarboxylase transgene expression \& analysis of environmental stress tolerance in transgenic rice”, Plant Sci, vol. 160, pp.869-75, 2001. 
[69] Cheng L., Zou Y., Ding S., Zhang J., Yu X., Cao J., and Lu G., "Polyamine accumulation in transgenic tomato enhances the tolerance to high temperature stress", J Integer Plant Biol, vol. 51, pp.489-499, 2009.

[70] Wen X-P., Pang X-M., Matsuda N., Kita M., Inow H., Hao Y-J., Honda C., and Moriguchi T., "Overexpression of the apple spermidine synthase gene in pear confers multiple abiotic stress tolerance by altering polyamine titers", Transgenic Res, vol.17, pp.251-263, 2008. 